\title{
ON SINGULARITIES OF SURFACES IN $E^{4}$
}

\author{
BY JOHN A. LITTLE
}

Communicated by Raoul Bott, July 29, 1968

1. Notation. Let $f: M^{2} \rightarrow E^{4}$ be an immersion of a compact orientable surface. Let $e_{1} e_{2} e_{3} e_{4}$ be orthonormal righthanded frames, $e_{1} e_{2}$ tangent and agreeing with a fixed orientation of $M$. As usual define $\omega_{i}$ and $\omega_{i j}$ by

$$
d f=\sum \omega_{i} e_{i} \quad d e_{i}=\sum \omega_{i j} e_{j}, \quad i=1, \cdots, 4 .
$$

The connection forms in the tangent and normal bundles are respectively $\omega_{12}$ and $\omega_{34}$. The respective curvature forms are $d \omega_{12}$ and $d \omega_{34}$. The Gauss curvature $K$ and the normal curvature $N$ satisfy (and may be defined by)

$$
d \omega_{12}=-K \omega_{1} \wedge \omega_{2}, \quad d \omega_{34}=-N \omega_{1} \wedge \omega_{2} .
$$

\section{Statement of the main results.}

THEOREM 1. Suppose $f: M \rightarrow E^{4}$ is an immersion such that $N$ is everywhere positive (negative). Then

$$
\chi(N M)=-2 \chi(M) \quad(\chi(N M)=2 \chi(M)) .
$$

Here $\chi(N M)$ is the Euler characteristic of the normal bundle and $\chi(M)$ is the Euler characteristic of $M$.

CoROllary 2. Every immersion of the sphere or torus must have a point where $N=0$.

The proof of Theorem 1 uses a geometrically defined field of tangent axes. In order to define these axes we review some of the local theory of surfaces in $E^{4}$.

3. The curvature ellipse [1]. The local invariants of a surface in $E^{4}$ are characterized by an ellipse in the normal plane. To define this ellipse let us first define a map $\eta: S_{p} \rightarrow N_{p}, S_{p}$ is the unit tangent circle at $p$ and $N_{p}$ is the normal plane at $p$. Let $\gamma(s)$ be a geodesic of $M$ through $p$ such that $d \gamma / d s(p)=e_{1}$, where $e_{1}$ is a unit vector at $p$. Define $\eta$ by $\eta\left(e_{1}\right)=d^{2} \gamma / d s^{2}(p)$. The curvature ellipse is the image of $S_{p}$ under $\eta$.

The mean curvature vector $\mathfrak{H C}$ is the position vector of the center of this ellipse. 
4. Construction of a field of axes [1]. In general the line through the mean curvature vector meets the curvature ellipse in two diametrical points. The inverse image under $\eta$ of these two points are four unit tangent vectors which form a pair of orthogonal tangent lines, i.e. an axis. This construction fails only when $\mathfrak{H}=0$ or at an inflection point.

THEOREM 3. The singular locus (inflection points and points where $\mathfrak{H}=0$ ) of the field of axes constructed above is generically a set of isolated points. The index is generically $\pm \frac{1}{2}$.

5. Sketch of the proof of Theorem 1. If $N>0$ then the point cannot be an inflection point. Thus the field of axes constructed above has singularities only at points where $\mathfrak{H C}=0$. These are generically isolated. Let them be $p_{1} \cdots p_{n}$. Let $\operatorname{Ind}_{1}\left(p_{i}\right)$ be the index of this field of axes at $p_{i}$. Generically $\operatorname{Ind}_{1}\left(p_{i}\right)= \pm \frac{1}{2}$. On the other hand the mean curvature vector is a normal vector and hence gives a normal vector field with singularities at $p_{1}, \cdots, p_{n}$. Let $\operatorname{Ind}_{2}\left(p_{i}\right)$ be the index of $\mathfrak{H C}$ at $p_{i}$. Generically $\operatorname{Ind}_{2}\left(p_{i}\right)= \pm 1$. The proof then consists in showing that if $N>0$ then $\operatorname{Ind}_{1}\left(p_{i}\right)$ and $\operatorname{Ind}_{2}\left(p_{i}\right)$ have opposite signs (and if $N<0 \operatorname{Ind}_{1}\left(p_{i}\right)$ and $\operatorname{Ind}_{2}\left(p_{i}\right)$ have the same signs). Once this is established the proof follows readily from the fact that

$$
\chi(N M)=\sum \operatorname{Ind}_{2}\left(p_{i}\right), \quad \chi(M)=\sum \operatorname{Ind}_{1}\left(p_{i}\right) .
$$

\section{Proof of Corollary 2.}

$$
\chi(N M)=\frac{1}{2 \pi} \int_{M} N d A .
$$

Thus if $N>0(N<0)$ everywhere then $\chi(N M)>0(\chi(N M)<0)$. By Theorem 1 if $N>0$ (or $N<0$ ) everywhere then $\chi(M)<0$. Consequently we obtain a contradiction if $M$ is a torus or a sphere.

In the light of Theorem 1 it would be interesting to know of examples of immersions with everywhere positive $N$. We have not found any yet.

\section{REFERENCES}

1. C. L. E. Moore and E. B. Wilson, Differential geometry of two-dimensional surfaces in hyperspace, Proc. Amer. Acad. Arts and Sci. 52 (1916), 267-368.

2. J. Little, On singularities of submanifolds of higher dimensional Euclidean spaces, Thesis, University of Minnesota, Minneapolis, Minn., 1968.

University of Minnesota, Minneapolis, Minnesota 55455 